\title{
THE
}

$12-1982$

\section{Padé Phenomenology for Two-Body Bound States}

\author{
K. Hartt \\ University of Rhode Island, hartt@uri.edu
}

Follow this and additional works at: https://digitalcommons.uri.edu/phys_facpubs

Terms of Use

All rights reserved under copyright.

\section{Citation/Publisher Attribution}

Hartt, K. (1982). Padé phenomenology for two-body bound states. Physical Review C, 26(6), 2616-2619. doi: 10.1103/PhysRevC.26.2616

Available at: http://dx.doi.org/10.1103/PhysRevC.26.2616

This Article is brought to you for free and open access by the Physics at DigitalCommons@URI. It has been accepted for inclusion in Physics Faculty Publications by an authorized administrator of DigitalCommons@URI. For more information, please contact digitalcommons@etal.uri.edu. 


\title{
Padé phenomenology for two-body bound states
}

\author{
K. Hartt \\ Physics Department, University of Rhode Island, Kingston, Rhode Island 02881
}

(Received 9 August 1982)

\begin{abstract}
Padé approximants in the squared momentum variable, recently used for elastic scattering, are employed in generating accurate analytic approximants for bound states. Through iteration, $[L / L+1]$ approximants yield the lowest eigenstate of the homogeneous Lippmann-Schwinger equation for Yukawa, Malfliet-Tjon, and Reid soft core central potentials with, respectively, $L=1,2$, and 3 . Higher eigenstates are readily obtained; the second is given for the Yukawa potential. Analytic separable expansions and scattering expressions result.
\end{abstract}

$\left[\begin{array}{c}\text { NUCLEAR STRUCTURE Padé approximants in } k^{2} \text {, analytic two- } \\ \text { body bound states, separable expansions, effective range parameters. }\end{array}\right]$

Two-body bound states, most notably the deuteron, have long been the subject of analytic investigations. However, analytic methods have met with limited success. Although the Rayleigh-Ritz method has proved useful, ${ }^{1}$ as have iterative techniques, ${ }^{2}$ the former leaves unsolved the important problem of basis optimization, while the latter can lead to rapid growth of complexity with each iteration. Even though in the computer era the twobody problem no longer demands analytic solutions, they are patently desirable because of their tractability and their possible use as stepping stones to solutions of few-body problems. For example, their use in constructing separable representations of potentials ${ }^{3}$ can have a decisive advantage in three $e^{4,5}$ and four ${ }^{6}$ body problems. In this paper we introduce a new method of using Padé approximants (PA) (Ref. 7) for iteratively generating analytic solutions of two-body bound-state problems that is accurate and simple to use, and we use this method to construct analytic separable expansions and expressions for effective range parameters.

A PA is defined as a rational function approximant, with its coefficients determined by equating the truncated Taylor expansions of the PA and the function being approximated. Previous work has shown reasonable analytic approximants in momentum space to possess a simple pole structure such as naturally arises from using PA. ${ }^{4}$ Also, it has recently been found that $x \equiv k^{2}$ is a natural variable for PA to the two-body scattering function, $F(x)=k \cot \left(\delta_{0}\right)$, associated with realistic $N N$ potentials, ${ }^{8}$ and that such approximants closely reproduce exact results. The bound state results we give here from using PA also yield rational scattering functions when employed with the unitary pole approximation (UPA) (Ref. 9) or the unitary pole expansion (UPE). ${ }^{10}$ When applicable, the present method appears to be a powerful alternative to previous uses of PA's for solving the integral equations of fewbody systems. ${ }^{7}$

The present method consists of assuming a Padé form for the solution of a two-body bound state problem and recovering that same form from a series expansion of the integral equation. Unlike the Thomas-Sachs method, ${ }^{2}$ ours leaves the complexity of our eigenfunction unchanged during the iteration process. We consider the case where the potential is a sum of Yukawas; for numerical results we further specialize to a single Yukawa which models the triplet $n p$ interaction $(\mathrm{Y}),{ }^{5}$ the ${ }^{1} S_{0}$ Malfliet-Tjon potential (MT), ${ }^{11}$ which is a sum of two Yukawas, and the ${ }^{1} S_{0}$ Reid soft core potential (RSC), ${ }^{12}$ which is a sum of three Yukawas. The last two, especially the RSC, have strong short-range repulsions. For $\mathrm{Y}, B=2.240 \mathrm{MeV}$; for $\mathrm{MT}$ and RSC, we set the binding energy $B$ to 0 , following Harms. $^{10}$

The potential is written:

$$
V(r)=\sum_{j=1}^{n} V_{j} \frac{\exp \left(-\mu_{j} r\right)}{\mu_{j} r} .
$$

The two-body Schrödinger equation for a bound $S$ state,

$$
\psi(k)=\left[E-\frac{k^{2}}{M}\right]^{-1} \int_{0}^{\infty} d y y^{2} V_{0}(k, y) \psi(y),
$$


when written with the potential of Eq. (1) in the momentum representation, becomes

$$
\begin{aligned}
\chi(k)=\lambda \sum_{j=1}^{n} \frac{g_{j}}{2 \pi} \int_{-\infty}^{\infty} & d y \frac{y}{k} \frac{\chi(y)}{y^{2}+\gamma_{0}^{2}} \\
& \times \ln \left(\mu_{j}^{2}+(y+k)^{2}\right),
\end{aligned}
$$

where we use natural units $(\hbar=c=1)$, we define strengths $g_{j}=-M V_{j} / \mu_{j}(M / 2$ is the reduced mass) and $\gamma_{0}{ }^{2}=M B$ ( $B$ is the binding energy), and we have multiplied out the bound state pole:

$$
\chi(k)=\left(k^{2}+\gamma_{0}^{2}\right) \psi(k) .
$$

The overall strength parameter $\lambda$ is introduced as an eigenvalue and equals unity if the potential produces the binding energy $E=-B$. Our equation for $\chi(k)$ is identical to the homogeneous Lippmann-Schwinger equation used by Harms to generate the UPE, and $\chi(k)$ becomes the form factor for the UPA when $\lambda=1$ :

$$
V_{0}\left(k^{\prime}, k\right)=-\left\langle k^{\prime} \mid \chi\right\rangle\langle\chi \mid k\rangle,
$$

with $\chi(k) \equiv\langle k \mid \chi\rangle$. The UPE is given by

$$
V_{0}\left(k^{\prime}, k\right)=-\sum_{j=1}^{n} \frac{\left\langle k^{\prime} \mid \chi_{j}\right\rangle\left\langle\chi_{j} \mid k\right\rangle}{\lambda_{j}},
$$

where $\chi_{j}(k)$ are eigenfunctions of the LippmannSchwinger kernel with $M E=-\gamma_{0}{ }^{2}$ and having eigenvalues $\lambda_{j}$. Orthonormality holds in the form

$$
\left\langle\chi_{n}\left|G_{0}(-B)\right| \chi_{m}\right\rangle=-\delta_{n m}
$$

where $G_{0}(E)=\left(E-k^{2} / M\right)^{-1}$

The solutions $\chi_{n}(k)$ and $\lambda_{n}$ are expressible, for $E<0$, in terms of an eigenvalue problem for a Hermitian operator, $G_{0}^{1 / 2} V G_{0}^{1 / 2} \cdot{ }^{13}$ Consequently, properties of the spectrum are readily obtained and the Rayleigh-Ritz method produces variational bounds on eigenvalues.

We write the even function $\chi(k)$ as a PA in the variable $x \equiv k^{2}$ :

$$
\chi(k)=\frac{P_{L}(x)}{Q_{M}(x)} \equiv[L / M],
$$

where $L$ and $M$ are the degrees of the polynomials $P_{L}$ and $Q_{M}$, and we choose $M=L+1$, which assures normalizability. The Hulthén function obtained by $\mathrm{Sachs}^{2}$ has a [0/1] structure. Anticipating our result that the stable solutions have poles on the positive imaginary axis, we obtain the equation

$$
\chi(k)=\lambda \sum_{p=1}^{n} \sum_{q=1}^{m} \frac{g_{p}}{k}\left[h_{q} \tan ^{-1}\left(\frac{k}{d_{p q}}\right)\right],
$$

where $m-1$ is the number of poles of $\chi(k)$ on the positive imaginary axis. The constants $h_{q}$ and $d_{p q}$ are simply and analytically expressible in terms of the Padé coefficients and $\gamma_{0}$. A PA for $\chi(k)$ is recovered and a value of $\lambda$ obtained using the first $2 L+2$ terms of the series expansion of the right hand side of Eq. (5). The solution, found iteratively by using the recovered $\chi(k)$ as an input to the right hand side, generally requires about 30 iterations to get 14-figure convergence of all parameters. Table I gives our results for the three potential models, showing the lowest order PA required for convergence. In coordinate space the wave functions are sums of exponentials, all range parameters being uniquely determined by our method, while in momentum space the wave functions closely resemble those previously given., ${ }^{40,14}$ The Yukawa model used by Harms ${ }^{14}$ differs so slightly from the one we employ ${ }^{5}$ that comparisons to three figures can still be made. It is also possible to obtain binding energies iteratively for those potentials that have bound states, by varying $B$ until $\lambda$ equals unity. For our Yukawa model, this yields $B=2.202 \mathrm{MeV}$, producing the characteristically larger error in $B$ $(1.7 \%)$ than in $\lambda(0.27 \%)$.

Higher eigenstates can also be obtained either iteratively or by diagonalization, as will be discussed in more detail in a forthcoming paper. The natural basis for either approach is the $L+1 \mathrm{di}$ mensional subspace spanned by the $[L / L+1]$ PA's possessing precisely the same denominator polynomials.

Table I reflects two approximations, the first being our basic ansatz that a PA form adequately represents the eigenfunction. Another approximation is made in the iteration procedure, by truncating the series expansion of the right hand side of Eq. (5). We have tested the ansatz by doing a Rayleigh-Ritz calculation with PA functions as a

TABLE I. First eigenvalue of Lippmann-Schwinger equation obtained through iteration compared with accurate results.

\begin{tabular}{cccc}
\hline \hline & & \multicolumn{2}{c}{ First } \\
Potential & PA & Iterative & Accurate \\
\hline Y & {$[1 / 2]$} & 1.003 & $1^{\mathrm{a}}$ \\
MT & {$[2 / 3]$} & -0.4419 & $-0.4342^{\mathrm{b}}$ \\
RSC & {$[3 / 4]$} & -0.05705 & $-0.06286^{\mathrm{c}}$ \\
\hline \hline
\end{tabular}

${ }^{a}$ Reference 5.

${ }^{b}$ Reference 10.

${ }^{\mathrm{c}}$ Reference 4. 
basis and using numerical quadrature. For simplicity, we shall restrict all further detailed discussion to the Yukawa model in the [1/2] approximation. Figure 1 shows the lowest eigenstate, $\chi_{1}(k)$, and the orthonormal [1/2] function, $\chi_{2}(k)$. They closely resemble the first two eigenfunctions numerically obtained for the Harms-Yukawa model. ${ }^{14}$ The Rayleigh-Ritz procedure is easily applied in the $\chi_{1}(k), \chi_{2}(k)$ subspace. For this purpose 20-point Gauss-Legendre integration is sufficiently accurate to compute matrix elements. If we write

$$
|\chi\rangle=\alpha\left|\chi_{1}\right\rangle+\beta\left|\chi_{2}\right\rangle
$$

then solving the equations

$$
\left\langle\chi_{1}\left|G_{0}(-B) A\right| \chi\right\rangle=\lambda\left\langle\chi_{i}\left|G_{0}(-B)\right| \chi\right\rangle,
$$

$i=1,2$, with $A=V G_{0}(-B)$ and the orthonormality condition leads to the upper bounds $\lambda_{1} \leq 1.000036$ and $\lambda_{2} \leq 3.479074$. Our $\lambda_{2}$ for the Harms and Adhikari Yukawas are the same to three figures, compared with Harms's accurate value of 3.2846. We obtain a small admixture $\beta=0.606128 \times 10^{-2}$ of $\left|\chi_{2}\right\rangle$ to the first eigenstate. Hence, $G_{0}(-B) A$ is practically diagonal in the Padé-iterated basis, and the plots of the new approximate eigenfunctions are indistinguishable from those in Fig. 1. Varying $B$, we obtain $B=2.2395$ for $\lambda=1$, which is in striking agreement, differing by 0.02 percent from the exact binding energy. These results strengthen the credibility of our approach for generating analytic solutions introduced in this paper.

With our $\left|\chi_{1}\right\rangle$ and $\left|\chi_{2}\right\rangle$, analytic approximations to the rank-1 UPA and the rank-2 UPE are

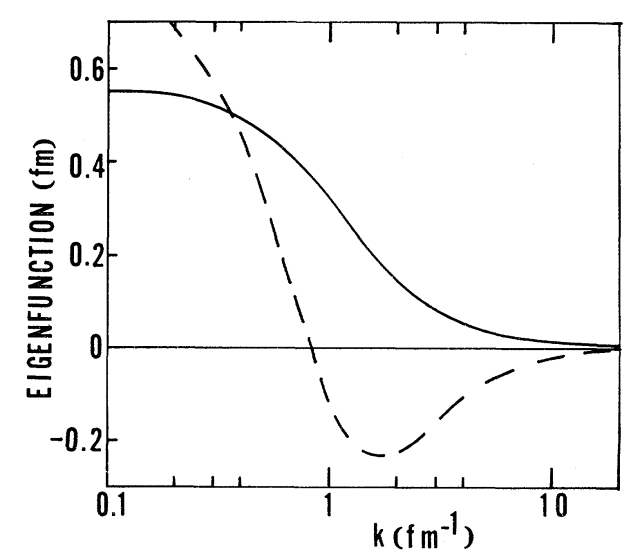

FIG. 1. Iterative eigenfunction $\chi_{1}(k)$ in [1/2] approximation (solid line) and $\chi_{2}(k)$ (dashed line) obtained by orthogonalizing to $\chi_{1}(k)$ while maintaining the same Padé denominator, $Q_{2}\left(k^{2}\right)$. Both functions are normalized. immediately obtained, and the scattering function $F\left(k^{2}\right)=k \cot \left(\delta_{0}\right)$ is easily found from the $R$ matrix. The $R$-matrix integrals can all be evaluated analytically and lead to rational forms for $F\left(k^{2}\right)$. For the present Yukawa potential, convergence of the UPE series contributions to $F\left(k^{2}\right)$ is known to be slow, ${ }^{5}$ although it is much faster for potentials with short-range repulsions. ${ }^{4,10}$ We limit our discussion here to the scattering length. We define the integral

$$
I\left(\gamma^{2}\right)=M \int_{0}^{\infty} \frac{d y y^{2} \chi_{1}{ }^{2}(y)}{\gamma^{2}+y^{2}} .
$$

By the normalization condition, $I\left(\gamma_{0}{ }^{2}\right)=1$. The UPA scattering length is

$$
a=\frac{\pi M \chi_{1}^{2}(0)}{2\left(I(0)-\lambda_{1}\right)},
$$

and if we set $\lambda_{1}=1$ we guarantee that the $T$ matrix will have the correct bound state pole, and we obtain $a=5.614 \mathrm{fm}$ compared with the more precise value of $5.470 \mathrm{fm}$ that we computed from the Schrödinger equation in coordinate space. The numerical result just given comes from the use of the iterative $\chi_{1}(k)$ and $\chi_{2}(k)$. Scattering length calculations reported here are all insensitive to choice of basis, whether iterative or Rayleigh-Ritz. Our two-term UPE leads to a scattering length of the form

$$
a=\frac{a_{1}+a_{2}-2 a_{1} a_{2} J(0)}{1-a_{1} a_{2} J^{2}(0)},
$$

where $a_{1}$ and $a_{2}$ are the scattering lengths associated with the two separable terms in $V$ and

$$
\begin{aligned}
& \pi(M / 2) \chi_{1}(k) \chi_{2}(k) J\left(k^{2}\right) \\
& =P \int_{0}^{\infty} d y y^{2} \chi_{1}(y) \chi_{2}(y) G_{0}\left(\frac{k^{2}}{M}\right) .
\end{aligned}
$$

The weakly attractive second term in $V$ associated with $\chi_{2}(k)$ drives the total scattering length to a value of $-0.7 \mathrm{fm}$ when Rayleigh-Ritz eigenvalues are used. If $\lambda_{1}$ and $\lambda_{2}$ are allowed to vary, a better fit to low energy scattering is achieved at the cost of shifting the bound state pole in the $T$ matrix. If $\lambda_{1}=1$ is used, the bound state pole is reproduced, but the second term in $V$ must be strongly repressed $\left(\lambda^{2} \simeq=110\right)$ to fit the scattering length to the quoted precision.

Although these results are approximations to a UPE analysis, the simple analytic structures permit extensions with great ease. Under current investigation is the possibility of fitting the bound state pole and the scattering function $F\left(k^{2}\right)$ at low energies 
with the use of several terms in a separable expansion for $V$, by varying all the $\lambda$ except for $\lambda_{1}$ which is determined by the bound state pole. Two other problems that would require some generalization of the present method are the deuteron with tensor forces and the triton with finite rank potentials. Both systems are reducible to coupled integral equations in one variable. We are investigating the possibility that they can be solved analytically in a similar manner.
We wish to thank George A. Baker, Jr., for discussions and some important suggestions, Jill C. Bonner for encouragement and useful comments, and P. V. A. Yidana for assistance in checking analytic expressions. We acknowledge the hospitality of Los Alamos National Laboratory where this work was initiated, computer time provided by the University of Rhode Island Academic Computer Center, and partial support through a URI research grant-in-aid.
${ }^{1}$ L. Hulthén and K. Laurikainen, Rev. Mod. Phys. 23, 1 (1951).

${ }^{2}$ R. Sachs, Nuclear Theory (Addison-Wesley, Cambridge, Mass., 1953).

3J. Levinger, in Springer Tracts in Modern Physics, edited by G.Höhler (Springer, Berlin, 1974), Vol. 71.

${ }^{4}$ E. Harms and L. Laroze, Nucl. Phys. A160, 449 (1971).

${ }^{5}$ S. Adhikari, Phys. Rev. C 10 , 1623 (1974).

${ }^{6}$ B. Gibson and D. Lehman, Phys. Rev. C 23, 404 (1981).

${ }^{7}$ G. Baker, Jr., Essentials of Padé Approximants
(Academic, New York, 1975).

${ }^{8}$ K. Hartt, Phys. Rev. C $\underline{22}, 1377$ (1980); 23, 2399 (1981).

${ }^{9}$ M. Fuda, Nucl. Phys. A116, 83 (1968).

${ }^{10}$ E. Harms, Phys. Rev. C $\underline{1}, 1667$ (1970).

${ }^{11}$ R. Malfliet and J. Tjon, Nucl. Phys. A127, 161 (1969).

12R. Reid, Ann. Phys. (N.Y.) 50, 411 (1968).

${ }^{13}$ S. Weinberg, Phys. Rev. 131, 440 (1963).

${ }^{14}$ E. Harms, Ph.d. thesis, Rensselaer Polytechnic Institute, 1969 (unpublished). 